Check for updates

Cite this: RSC Adv., 2018, 8, 24525

\title{
Potential use of kraft and organosolv lignins as a natural additive for healthcare products $\dagger$
}

\author{
Oihana Gordobil, ${ }^{a}$ René Herrera, ${ }^{a}$ Marwa Yahyaoui, ${ }^{\text {ab }}$ Sedef ilk, ${ }^{c}$ Murat Kaya (D) d \\ and Jalel Labidi $\mathbb{D}$ *a
}

The growing interest in substituting synthetic products coming from non-renewable sources with products from biomass has focused attention on the lignin biopolymer. Its high availability, low price and properties make the development of new and valuable uses for lignin interesting, thus improving the economic and environmental aspects of the biomass conversion. To achieve this objective, the potential use of industrial kraft and organosolv lignins as antioxidants, antimicrobials and sunscreen products has been evaluated. The results of a detailed antibacterial and antifungal study demonstrated the high potential of kraft lignins against a variety of foodborne and human pathogenic microorganisms. Moreover, both organosolv and kraft lignins presented an effective protection factor (SPF values from 10-20), demonstrating their effectiveness as natural additives for the sun lotion market. In addition, lignin samples presented high antioxidant capacity compared to butylated hydroxytoluene (BHT), one common commercial antioxidant industrially used. Therefore, the development of innovative applications of lignins as a commodity for the chemical, pharmaceutical or cosmetic industries could expand their possible uses in the market giving new added values to lignin.

Received 14th March 2018

Accepted 30th June 2018

DOI: $10.1039 / \mathrm{c} 8 \mathrm{ra} 02255 \mathrm{k}$

rsc.li/rsc-advances wood fibers to elaborate pulp and paper, large quantities of black liquor are generated annually as a by-product. This black liquor contains dissolved lignin, which can be isolated by precipitation obtaining a sulfur containing lignin better known as kraft lignin. Eucalyptus and spruce are widely used feedstocks for pulp and paper manufacture because of their high cellulose content and fast growth. In addition, the organosolv method, in which organic compounds such as alcohols and organic acids are mainly used, has attracted increasing attention in recent years due to being environmentally friendly as well as offering less degraded and sulphur free lignin with high purity. $^{2-5}$

The main physical and biological functions of lignins are conferring structural support to the plant, participating in internal transport of water and nutrients and providing protection against chemical and biological attack. ${ }^{6}$ Moreover, plants with higher lignin content have been reported to be more resistant to direct sunlight. ${ }^{7}$ These natural properties, in addition to the industrial abundance of this aromatic compound, make the lignin a very attractive and versatile compound to replace synthetic products, thus giving a new value added product to biomass conversion improving the economic profit of the industry.

The high potential of lignin as antioxidant product have been already reported by previous works ${ }^{8-11}$ making it as good alternative to replace cytotoxic synthetic antioxidants like butylated hydroxytoluene (BHT) or butylated hydroxyanisole (BHA), which are widely used in industries such as $\dagger$ Electronic supplementary information (ESI) available. See DOI: $10.1039 / \mathrm{c} 8 \mathrm{ra} 02255 \mathrm{k}$
${ }^{a}$ Chemical and Environmental Engineering Department, University of the Basque labidi@ehu.eus

${ }^{b}$ Laboratory of Materials and Molecules Application, University of Tunisia El Manar, Farhat Hached University Campus PB 94 - Rommana 1068, Tunis, Tunisia

'Ömer Halisdemir University, Central Research Laboratory, TR-51240, Niğde, Turkey ${ }^{d}$ Department of Biotechnology and Molecular Biology, Faculty of Science and Letters, Aksaray University, Aksaray, Turkey 
pharmaceuticals, cosmetic, food and plastics. The urge to find alternatives to synthetic antioxidants lies on the fact that several studies have proved their harmful effect at molecular and cellular levels, even at low concentrations. ${ }^{\mathbf{1 2 - 1 5}}$ Moreover, lignin polymer has also proven its inhibitory effect against some microorganisms such as bacteria and fungus. ${ }^{12,16}$ However, their inhibitory capacity depends mainly on the genetic origin of the lignin as well as the isolation process.

Nowadays, another issue of great concern is the damaging effect of the sun. Solar ultraviolet radiations are divided into three categories: UVC (200-280 nm), UVB (280-320 nm) and UVA (320-400 nm). ${ }^{17}$ The most biologically harmful radiation is UVC, but it is filtered out by ozone layer. UVA has accumulated effect causing premature skin aging and UBV is the main responsible of skin sunburn and skin cancer. ${ }^{18-20}$ Therefore, the use of sunscreens with ability to block UVB is very important to prevent the negative effects of sun exposure. Chemical and physical sunscreens are used to protect our skin against sun and the most usual active ingredients are homosalate and avobenzone for chemical sunscreen lotions and zinc oxide and titanium dioxide for physical sunscreens creams. ${ }^{\mathbf{1 9 2 1}}$ Nevertheless, long-time use of such commercial creams may cause a health risk to the skin. Therefore, natural sunscreens are receiving an increasing attention; several natural products have been already tested to replace the chemical and physical sunscreen additives by using herbal oils and extracts. ${ }^{18,22}$ In this context, lignin molecule could offer a great contribution to the sunscreen field. Due to the chemical structure of lignin molecule, it could act as sun blocker helping to the replacement of synthetic sunscreen product used nowadays. Moreover, recent publications have proved the non-toxicity of lignin molecule for human tissues ${ }^{9}$ making them suitable for cosmetic industry.

This work offers a comparative study of extracted organosolv lignins from spruce and eucalyptus with their analogous kraft lignins isolated from the industry. The main objective was to evaluate selective functional properties of different lignin samples in order to explore their potential use for different fields. For this purpose, antioxidant potential was analyzed by two common used methods; antifungal capacity against Aspergillus niger and antibacterial capacity against 9 foodborne and human pathogenic microorganisms were assessed. Finally, their potential as natural additive for sunscreen was evaluated.

\section{Materials and methods}

\subsection{Materials}

Lignin samples (OS: organosolv spruce; KS: kraft spruce; OE organosolv eucalyptus; KE: kraft eucalyptus) were obtained according to methods described in a previous work. ${ }^{23}$ The reagents used for this work were: dimethyl sulfoxide (DMSO, PanReac), methanol HPLC grade (Fisher), gallic acid monohydrate (Scharlau), 2,2-diphenyl-1-picrylhydrazyl (DPPH, SigmaAldrich), 2,2'-azino-bis3-ethylbenzothiazoline-6-sulphonic acid (ABTS, Sigma-Aldrich), BHT (Sigma-Aldrich), Trolox 97\% (Sigma-Aldrich), potato dextrose agar (PDA, Scharlau).

\subsection{Determination of phenolic content}

The total phenolic content (TPC) of lignin samples was determined by the Folin-Ciocalteau spectrophotometric method using gallic acid as reference compound and dimethyl sulfoxide as solvent. As first step, a calibration curve using gallic acid as reference in DMSO was calculated with six different concentrations (100-1000 $\left.\mathrm{mg} \mathrm{L}^{-1}\right)$. Lignin samples were dissolved in DMSO $\left(2 \mathrm{~g} \mathrm{~L}^{-1}\right) . \mathrm{Na}_{2} \mathrm{CO}_{3}$ at $200 \mathrm{~g} \mathrm{~L}^{-1}$ was prepared. For the analysis, $0.5 \mathrm{~mL}$ of lignin solution, $2.5 \mathrm{~mL}$ of Folin-Ciolcalteau reagent and $5 \mathrm{~mL}$ of $\mathrm{Na}_{2} \mathrm{CO}_{3}$ were added to $50 \mathrm{~mL}$ flask and covered with distilled water. The samples were kept in a thermostatic bath at $40{ }^{\circ} \mathrm{C}$ for $30 \mathrm{~min}$ before spectrophotometric measurement of the absorbance at $750 \mathrm{~nm}$ (Jasco V-630 spectrophotometer). The blank was prepared in the same way but adding $0.5 \mathrm{~mL}$ of DMSO instead of sample. The total phenolic content of lignin samples was expressed as the percentage gallic acid equivalents (mg GAE/g lignin) and hydroxyls content (wt\%). Both parameters were calculated on dry basis.

$$
\begin{gathered}
\operatorname{GAE}(\%)=\frac{C_{\mathrm{GAE}}}{C_{\text {sample }}\left[1-\frac{(H)}{100}\right]} \times 100 \\
\mathrm{OH}(\%)=\frac{C_{\mathrm{GAE}}}{170.12 \times 1000} \times 4 \times 17 \times \frac{1}{C_{\text {sample }}}
\end{gathered}
$$

where $C_{\mathrm{GAE}}$ is the concentration of gallic acid obtained by the calibration curve, $C_{\text {sample }}$ is the concentration of the lignin sample in DMSO (expressed as $\mathrm{mg} \mathrm{L}^{-1}$ ) and $H$ is the humidity content.

\subsection{Determination of antioxidant capacity}

The antioxidant activity of samples should be determined by various methods. DMSO was used to dissolve the lignin samples at different concentrations $\left(0-2 \mathrm{mg} \mathrm{mL}^{-1}\right)$. The DPPH scavenging activity was evaluated using the method described by Brand-Williams et al., 1995 (ref. 24) with some modifications. $0.1 \mathrm{~mL}$ of lignin solution was added to $3.9 \mathrm{~mL} \mathrm{DPPH}\left(25 \mathrm{mg} \mathrm{L}^{-1}\right.$ in methanol). The absorbance was measured at $517 \mathrm{~nm}$ after 30 min of incubation at room temperature. For ABTS assay, a radical solution (7 $\mathrm{mM}$ ABTS and $2.45 \mathrm{mM}$ potassium persulphate) was prepared and left to stand in the dark at room temperature for $16 \mathrm{~h}$ before using. This solution was then diluted with ethanol to an absorbance of $0.70 \pm 0.02$ at $734 \mathrm{~nm}$ and equilibrated at $30{ }^{\circ} \mathrm{C}$. For the analysis, $2 \mathrm{~mL}$ of the diluted radical solution were mixed with $20 \mu \mathrm{L}$ of the sample and the absorbance at $734 \mathrm{~nm}$ was read against ethanol in a Jasco V-630 spectrophotometer. The absorbance at $734 \mathrm{~nm}$ of all the samples was registered after 6 minutes. In both analyses, commercial antioxidants (BHT and Trolox) were used as positive control. Each test was carried out in triplicate. The inhibition percentage of the DPPH and ABTS radical was calculated according to the following formula:

$$
\text { Inhibition }(\%)=\frac{A_{0}-A_{1}}{A_{0}} \times 100
$$


where $A_{0}$ is the absorbance of control, and $A_{1}$ is the absorbance of lignin sample. The radical scavenging activity of the lignin was characterized using the term of "Efficient Concentration" or $\mathrm{IC}_{50}$, which is the concentration required for $50 \%$ inhibition of the free radical. Radical-scavenging activity was plotted as a function of the lignin concentration in the radical system.

\subsection{Antimicrobial bioassay of lignin compounds}

For antifungal activity of lignins determination, lignin samples (OS, KS, OE, KE) were diluted in DMSO at different concentrations $(1 \%, 2.5 \%, 5 \%, 7.5 \%, 10 \%, 15 \%$ and $20 \%)$ and then was evaluated the antifungal activity against A. niger (Tiegh MB284309 CBS-KNAW, Holland) by impregnation and exposition to fungal strain in PDA. Cellulose disc $(\varnothing=10 \mathrm{~mm})$ were soaked with $40 \mu \mathrm{L}$ of each lignin solution and placed on Petri dishes with PDA $(10 \mathrm{~mL})$ and $0.4 \%$ streptomycin (antibacterial agent). Each PDA dish was inoculated with fungal spores of $A$. niger, and incubated for 7 days at $27 \pm 1.5^{\circ} \mathrm{C}$. Subsequently, the disc were removed from Petri dishes and washed with sterile Ringer's solution (Sigma-Aldrich-96724). DMSO was used as control. The solution was stained (trypan blue) and homogenized to count the spores concentration on the pellets with a Cellometer ${ }^{\circledR}$ Mini (Nexcelom Bioscience LLC) automated cell counter by putting $20 \mu \mathrm{L}$ of each spore solution inside counting chambers and using Cellometer ${ }^{\circledR}$ Mini software for the analysis. The fungal growth inhibition (FGI \%) was calculated as concentration of spores (conidia) per millilitre according to the following equation:

$$
\mathrm{FGI}(\%)=\frac{C_{\mathrm{g}}-T_{\mathrm{g}}}{C_{\mathrm{g}}} \times 100
$$

where $C_{\mathrm{g}}$ is the average concentration in the control sample and $T_{\mathrm{g}}$ is the average concentration in the treated one..$^{25}$

To analyze the antibacterial properties of lignin different bacteria cultures were used. Bacteria culture: food spoiling and human pathogen bacteria (Escherichia coli ATCC 25922, Staphylococcus aureus ATCC 25923, Proteus microbilis ATCC 14153, Proteus vulgaris ATCC13315, Pseudomonas aeruginosa ATCC 27853, Enterobacter aerogenes ATCC13048, Bacillus thuringiensis, Salmonella enterica serotype typhmurium SL 1344 and Streptococcus mutans ATCC 25175) were employed to evaluate the antibacterial properties of lignin samples (OS, KS, OE, KE). All bacteria strains were sub-cultured on (Luria Bertoni) LB agar culture at $37^{\circ} \mathrm{C}$ for $24 \mathrm{~h}$.

Disc-diffusion method (according to method of Clinical and Laboratory Standards Institute) was used for determination the antimicrobial properties of the lignin compounds against the food destroyed and human pathogen bacteria. For the disc diffusion assay, lignin samples $\left(0.02 \mathrm{~g} \mathrm{~mL}^{-1}, \mathrm{w} / \mathrm{v}\right)$ were dissolved in DMSO. Microorganisms' susceptibility was adjusted by 0.5 McFarland as a reference standard. The all prepared samples were sterilized under ultraviolet light for $5 \mathrm{~min}$ before test. Microorganism culture suspension $\left(100 \mu \mathrm{L}, 10^{6}\right.$ cells per $\mathrm{mL}$ ) was swabbed onto a plate within Müller-Hinton agar. Sterile discs $(\varnothing=6 \mathrm{~mm})$ were placed on the Petri plate inoculated with microorganisms and then, $20 \mu \mathrm{L}$ of the prepared lignin solution was loaded on the sterile discs. Finally, bacteria cultures were incubated at $37^{\circ} \mathrm{C}$ for $24 \mathrm{~h}$. The disc containing gentamicin (10 $\mu \mathrm{g}$ per disc) and only solvent (DMSO) were used as positive and negative control, respectively. All experiments were repeated triplicate. The results were expressed as mean diameter of inhibition zone in $\mathrm{mm} \pm$ standard deviation (mean $\pm \mathrm{SD})$.

\subsection{Determination of sun protection factor (SPF)}

Sun protection factor (SPF) of lignins was determined using in vitro screening method. It consists on measurement of UV radiation absorption through sunscreen product $(25 \mathrm{mg})$ added to quartz plates $(22 \times 22 \mathrm{~mm})$. Lignin-based sunscreen creams were prepared using DOVE body milk cream (Cream-D) and lignin at different concentrations $(1 \%$ and $5 \%)$ by magnetic stirring under room temperature in dark for $48 \mathrm{~h}$. In addition, DELIAL SPF 20 and 50 was used as positive control and DOVE body milk cream (Cream-D) was taken as negative control. Taking into account that UVB is 1000 times more erythemogenic than UVA, SPF indicates the protection against UVB. The absorbance values of each sample were determined using Jasco V-630 spectrophotometer from $290-320 \mathrm{~nm}$, at $5 \mathrm{~nm}$ intervals by using the following formula:

$$
\mathrm{SPF}=\mathrm{CF} x \sum_{290}^{320} \mathrm{EE}(\lambda) x I(\lambda) x \operatorname{Abs}(\lambda)
$$

where CF is correction factor (10), EE $(\lambda)$ is erythmogenic effect of radiation with wavelength $\lambda$, Abs $(\lambda)$ is spectrophotometric absorbance values at wavelength $\lambda$. The values of EE and $I$ are constants. They were determined by Sayre et al. 1979. ${ }^{26}$

\subsection{Color measurements}

The color of lignin was measured by PCE-CMS 7 colorimeter using the CIE-Lab color space coordinate system $L^{*}, a^{*}$, and $b^{*}$ (lightness, red-green-axis, and yellow-blue axis). For this work $L^{*}$ parameter was used to measured the lightness of each lignin sample (values from 0 to $100 \%$ ).

\subsection{Statistical analysis}

The results were analyzed by one-way analysis of variance (ANOVA) followed by Tukey and Bonferroni test considering significance level of $p<0.001, p<0.01$ and $p<0.5$. The software used for statistical analysis was Origin 8.6.

\section{Results and discussion}

Chemical and structural characterization of four lignin samples was determined in a previous work. ${ }^{23}$ The most important information about lignin samples was summarized in Table 1. The purity of lignins expressed as Klason (\%) showed high purity levels especially for OS (>90\%) followed by KS and OE. However, KE presented low purity due to its very high ash content $(>20 \%)$. Carbohydrates are usually the most known impurities in lignins; organosolv lignins presented higher sugar content than kraft lignins being OS the one with most 
Table 1 Purity, chemical structure parameters and molecular weight properties of lignins

\begin{tabular}{|c|c|c|c|c|}
\hline & OS & KS & $\mathrm{OE}$ & $\mathrm{KE}$ \\
\hline Klason (\%) & 94.3 & 88.5 & 83.7 & 58.6 \\
\hline ASL (\%) & 3.1 & 2.3 & 1.6 & 6.3 \\
\hline Carbohydrates (\%) ${ }^{a}$ & 9.7 & 2.7 & 4.1 & 2.9 \\
\hline $\mathrm{OCH}_{3}{ }^{b}$ & 0.7 & 0.85 & 1.19 & 1.14 \\
\hline $\mathrm{COOH}^{c}$ & 0.23 & 0.4 & 0.11 & 0.88 \\
\hline $\mathrm{Mn}^{d}$ & 1065 & 1540 & 1567 & 1059 \\
\hline $\mathrm{Mw}^{d}$ & 3081 & 7195 & 5079 & 2653 \\
\hline $\mathrm{IP}^{d}$ & 2.9 & 4.7 & 3.2 & 2.5 \\
\hline
\end{tabular}

${ }^{a}$ Determined by Py-GC/MS. ${ }^{b}$ Determined by ${ }^{13} \mathrm{C}$ NMR and expressed as functional groups per C9. ${ }^{c}$ Determined by ${ }^{31} \mathrm{P}$ NMR. ${ }^{d}$ Determined by gel permeation chromatography.

carbohydrate content. Another interesting compound to control is sulphur content. As expected, kraft lignins had sulfur containing compounds because of their origin from pulp and paper production process. Methoxyl content was determined by ${ }^{13} \mathrm{C}$ NMR being higher in eucalyptus lignins due to their high content in syringyl units as demonstrated $\mathrm{H} / \mathrm{G} / \mathrm{S}$ ratio obtained by Py-GC/MS technique. The lignin molecular weight average greatly depends on the feedstock and extraction process used. Therefore, as a result of different isolation conditions for each sample, no correlation could be made between the origin, isolation process and molecular weight properties of lignin.

\subsection{Determination of antioxidant activity and phenolic content}

ABTS and DPPH are simple indirect methods used for the evaluation of antioxidant potential of lignins. In both methods the radicals are reduced by the antioxidant, in this case lignin, and the color change is measured by spectrophotometric technique. ${ }^{27}$ In order to evaluate the inhibitory effect of lignin samples, BHT and Trolox were used as positive controls and the curves are shown in Fig. 1. Moreover, Table 2 presents efficient concentration $\left(\mathrm{IC}_{50}\right)$ and phenolic content of the lignin samples. As can be observed, the results of antioxidant activity of lignins did not show the same trend in ABTS and DPPH methods. These differences can be explained by the reaction mechanisms, in which ABTS radical reaction takes place at much faster rate due to electron transfer compared to DPPH. ${ }^{28}$ Moreover, Wang et al., 1998 (ref. 29) found that compounds which showed ABTS scavenging activity did not have DPPH scavenging activity. Statistical analysis of antioxidant results (Table 2) showed that lignins OS, KS and OE did not have statistically differences (Tukey and Bonferroni $p<0.001$ ) for $\mathrm{IC}_{50}$ value in $\mathrm{ABTS}$ method, indicating that $\mathrm{IC}_{50}$ value of $\mathrm{KE}$ lignin was significantly far from the other samples. In contrast, $\mathrm{IC}_{50}$ value in DPPH method was statistically different for all lignin samples (Tukey and Bonferroni $p<0.5$ ). For this reason, authors considered that DPPH method could be more accurate than ABTS for the analysis of antioxidant activity of lignins and the discussion was based on DPPH results. According to previous studies, the phenolic groups have a high influence on antioxidant activity of lignins., ${ }^{3,8}$ However, the radical power of phenolics not only depends on the formation of the phenoxyl radical but also on its stability. Therefore, substituents like methoxyl groups and conjugated double bonds can stabilize phenoxyl radicals by resonance providing a positive effect on antioxidant behavior of lignins., ${ }^{2,30}$ In addition, having low molecular weight and narrow polidispersity is also beneficial for antioxidant properties. ${ }^{9}$ Nevertheless, a conjugated carbonyl group has a negative effect on antioxidant activity, ${ }^{11}$ as well as impurities such as a high carbohydrate content, since the formation of hydrogen bonding with lignin block the formation
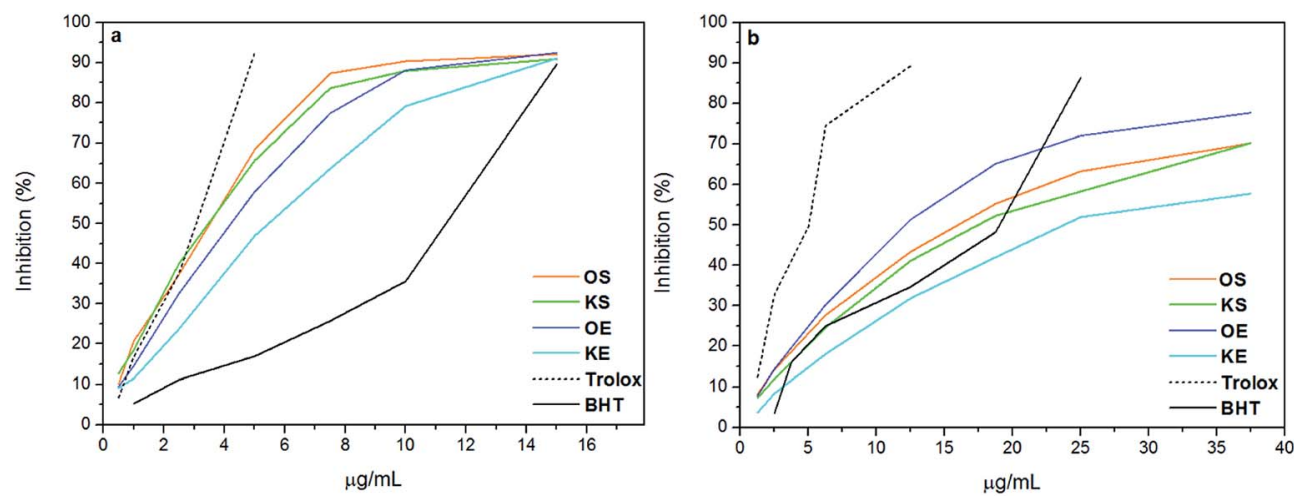

Fig. 1 Antioxidant activity of lignin against (a) ABTS and (b) DPPH and their comparison with BHT and Trolox used as positive controls. 
Table 2 Phenolic content and efficient concentration $\left(\mathrm{IC}_{50}\right)$ of lignin samples $^{a}$

\begin{tabular}{|c|c|c|c|c|}
\hline & & & ABTS* & $\mathrm{DPPH}^{* *}$ \\
\hline & GAE (\%) & $\mathrm{OH}(\mathrm{wt} \%)$ & $\mathrm{IC}_{50}$ & $\mathrm{IC}_{50}$ \\
\hline OS & 35.2 & 14.1 & $3.51 \pm 0.1$ & $15.85 \pm 0.3^{* *}$ \\
\hline KS & 33.5 & 13.4 & $3.47 \pm 0.4$ & $16.63 \pm 0.5^{* *}$ \\
\hline $\mathrm{OE}$ & 34.7 & 13.9 & $4.22 \pm 0.3$ & $12.85 \pm 0.9^{* *}$ \\
\hline $\mathrm{KE}$ & 22.8 & 9.1 & $5.46 \pm 0.2^{*}$ & $22.75 \pm 0.3^{* *}$ \\
\hline BHT & - & - & $10.8 \pm 0.8$ & $19.01 \pm 0.2$ \\
\hline Trolox & - & - & $2.80 \pm 0.0$ & $5.00 \pm 0.1$ \\
\hline
\end{tabular}

${ }^{a}$ Analysis of variance ANOVA: $p>0.001 ; p>0.5$ (Tukey and Bonferroni corrections: $^{*}=p>0.001$ and ${ }^{* *}=p>0.5$ ).

of free radical. ${ }^{5}$ In this study, the phenolic content of lignins, did not present a correlation between the origin and isolation process presenting similar total phenolic content for OS, KS and OE with values between 33-35 mg GAE/g lignin. However, KE had lower content of phenolic probably due to its low purity and high content of ashes. DPPH results showed a clear relation between lignin concentration and antioxidant activity, increasing the inhibitory effect at higher concentrations for all samples. The antioxidant activity of lignin samples decreased in the order OE $>$ OS > KS > KE. Further, it was observed that antioxidant activity of OS, KS and OE was higher than BHT control whereas it was lower than Trolox. However KE presented lower antioxidant capacity than BHT as shows Table 2. Similar results are found by other authors. ${ }^{11,31,32}$ Although OS, KS and OE showed similar phenolic content, the stronger DPPHscavenging capacity of $\mathrm{OE}$ was mainly due to the highest methoxyl content and lowest carboxylic acid content. On the other hand, the lower radical scavenging activity of KE could be partly attributed to the presence of very high content of carboxylic acids compared to other lignin samples as well as its low phenolic content together with its low purity. Concluding, the high antioxidant activity of the lignin samples, principally OS, KS and OE, suggested that they could act as potential antioxidant using for food, cosmetic and pharmaceutical industries instead of using BHT. In addition, recent publications have demonstrated the non-toxicity of lignin molecule for human tissues. ${ }^{9}$ Therefore, new opportunities to lignin compound in cosmetic industry would be interesting to develop.

\subsection{Antimicrobial activity of lignins}

For the determination of antifungal activity, Aspergillus niger was inoculated at different concentrations of lignin samples in order to know low, moderate and optimal potential inhibition effect of each lignin on cellulose pellets. Fig. 2 shows the results of this analysis where three efficiency ranges were included in order to establish a clear difference in fungal inhibition power of lignins. Inhibition values higher than $75 \%$ were taken as optimal inhibition, values between $50-75 \%$ as moderate inhibition and values lower than $50 \%$ as low inhibition. In general, both kraft lignins (KS and KE) presented higher inhibition potential than organosolv lignins (OS and $\mathrm{OE}$ ). KS act as

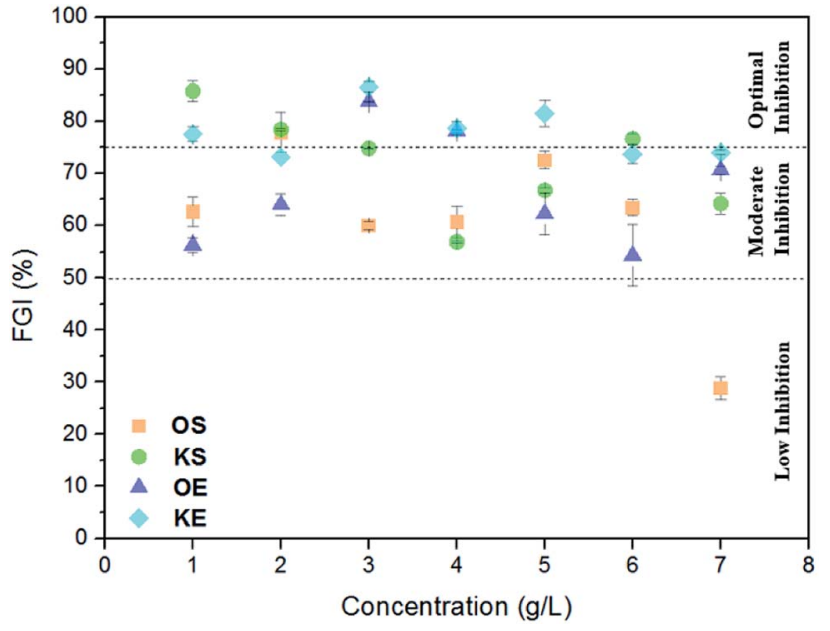

Fig. 2 Fungal growth inhibition (\%) of lignins.

antifungal product especially at low concentrations (up to 5\%) while moderate inhibition potential was found at high concentrations. However, KE presented a quite stable inhibition power at studied concentrations with inhibition values between 73-87\%. Organosolv lignin showed in general moderate inhibition effect. In addition, as can be observed, at high concentrations the inhibition effect decreased for all lignin samples, particularly in KS and OE $(r=-0.749$ and $r=-0.482$, respectively). Only few works have demonstrated that antifungal activity of lignin molecule depends mainly on the lignin origin and extraction method. ${ }^{12,33,34}$ Moreover, their composition and chemical structure has a vital importance like Zemek et al., 1979 (ref. 16) proved when studied different model compounds with guaiacyl and syringyl structure against some microorganism. Therefore, the higher inhibition power of kraft lignins could be due to their low carbohydrate content $(2.5-3 \%)$ compared to organosolv lignins $(4-10 \%)$, as well as the presence of sulfur containing derivatives as show Table 1 . Moreover, KE presented very high ash content $(\sim 23 \%)$ that could have an important influence on the antifungal inhibition effect since inorganic compounds usually are not desirable for fungus. ${ }^{35}$ To sum up, regarding the antifungal study the authors conclude that kraft lignins could be more suitable than organosolv lignins for application as antifungal product. Fig. 3 shows the antibacterial activity of obtained lignin samples against food and human pathogenic microorganisms. All the tested lignin samples exhibited greatly antibacterial affectivity against the studied bacteria. KS lignin displayed greater antimicrobial activity against E. coli ATCC $25922(17.72 \pm 1.16 \mathrm{~mm})$, P. microbilis ATCC $14153(19.12 \pm 1.22 \mathrm{~mm})$, P. vulgaris ATCC13315 (24.68 \pm $1.31 \mathrm{~mm})$, P. aeruginosa ATCC $27853(21.89 \pm 1.26 \mathrm{~mm}), E$. aerogenes ATCC13048 (18.97 $\pm 1.08 \mathrm{~mm})$, S. aureus ATCC 25923 $(19.18 \pm 0.98 \mathrm{~mm})$ and B. thuringiensis $(24.76 \pm 1.19 \mathrm{~mm})$ than tested KE lignin. In contrast, lignin of KE had higher antimicrobial property against microorganism S. typhmurium SL 1344 $(33.36 \pm 1.42 \mathrm{~mm})$ and S. mutans ATCC $25175(17.03 \pm 1.05$ $\mathrm{mm})$ than KS lignin. On the other hand, OS lignin showed greater antimicrobial activity against $E$. coli ATCC $25922(13.32$ $\pm 1.01 \mathrm{~mm})$, P. microbilis ATCC $14153(18.15 \pm 1.04 \mathrm{~mm}), P$. 


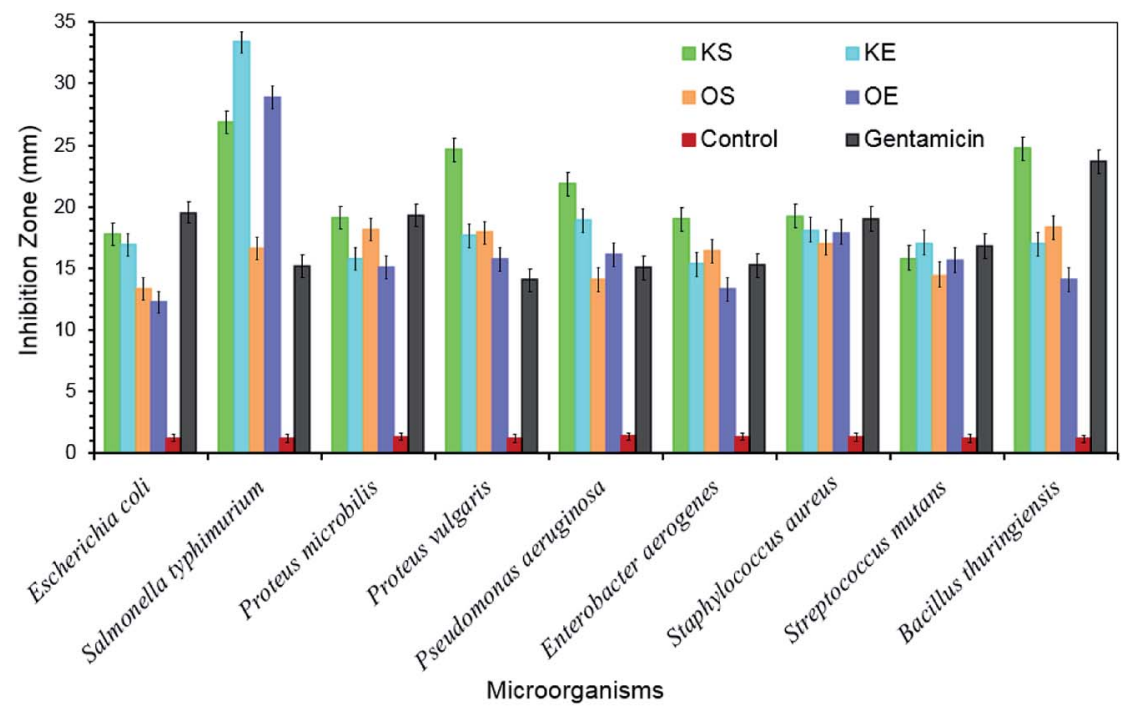

Fig. 3 Antimicrobial activities of lignin samples expressed as inhibition zone diameter (mm).

vulgaris ATCC13315 (17.91 $\pm 0.96 \mathrm{~mm})$, E. aerogenes ATCC13048 $(16.45 \pm 0.98 \mathrm{~mm})$ and $B$. thuringiensis $(18.36 \pm 1.09 \mathrm{~mm})$ than tested OE lignin sample. In addition, OE lignin exhibited higher activity against $S$. typhmurium SL $1344(28.92 \pm 1.36 \mathrm{~mm}), P$. aeruginosa ATCC $27853(16.10 \pm 1.06 \mathrm{~mm})$, S. aureus ATCC $25923(17.88 \pm 0.97 \mathrm{~mm})$ and $S$. mutans ATCC $25175(15.66 \pm$ $0.92 \mathrm{~mm}$ ) than OS lignin form. Thus, it was obtained that almost similar bacteria culture showed similar sensitivity against spruce lignins (KS and OS). In general, the antimicrobial activity of kraft lignins (KS and KE) had much higher than organosolv lignins (OS and OE). In all the tests, control disc with only solvent (DMSO) displayed slightly low (almost $1.2 \pm 0.06$ $\mathrm{mm}$ ) inhibition zone. Interestingly, the antimicrobial activity of the kraft lignins (KS and KE) was recorded higher than commonly used antibiotic (gentamicin) against almost all tested microorganism. This high antimicrobial activity of the lignin was thanks to its rich antioxidant and polyphenolic nature..$^{30,36,37}$ Therefore, lignin obtained in our study could be used effectively as an alternative natural antimicrobial additive or agent in food, textile or chemical industry against pathogenic microorganisms.

\subsection{Determination of in vitro SPF of lignin-based sunscreen lotions}

The in vitro SPF method is a useful quantitative test to check the effectiveness of the sunscreen product as a supplement of the in vivo measurements. In this research, lignin samples were blended with a standard commercial cream (Cream-D) at $1 \%$ and 5\% and their SPF values were determined using Mansur equation. ${ }^{\mathbf{1 0 , 1 2}}$ The SPF results of the controls and the creams containing lignins are shown in Table 3. The pure cream (Cream-D) did not show SPF, presenting values around 0.8. However, the measured SPF of the sunscreen lotion control (SPF 20) was $26.9 \pm 0.7$. This difference between theoretic SPF value and laboratory measured using the same evaluation method has been observed in previous works. ${ }^{21,38} \mathrm{SPF}$ evaluation proved that the addition of lignin in different concentrations to Cream-
D, has a great potential as sunscreen product. As expected, when lignin was added into pure cream (Cream-D), SPF values were increased. With the addition of only $1 \%$ to pure cream, SPF values of 10-12 were observed. Moreover, at 5\% of lignin higher SPF values were noticed, especially for OS and KE following by OE, while KS remained almost similar. Fig. 4 showed that pure cream (Cream-D) had no absorbance in UVA and UVB areas; however, cream with SPF 50 (cream-D50) exhibited transmittance of $0 \%$ demonstrating its high SPF. Higher lignin content decreased significantly the transmittance of elaborated creams. The excellent UV-radiation absorber property of lignin molecule is mainly due to its chemical structure with chromophores such as carbon-carbon double bonds, carbonyl groups and aromatic systems which affect on sun block action. ${ }^{38-40}$ In addition, auxochromes (hydroxyls groups and ethers) are important. ${ }^{19}$ Apart from the structure and functional groups, Gutiérrez-Hernández et al., 2016 (ref. 17) proved that the smaller size of lignin has a great positive effect in UV absorption properties of lignin. Moreover, Qian et al., 2016 (ref. 38) found that lignin with more methoxyl groups and carbonyl groups has better sunscreen performance as well as the sunscreens of lignin improved after ultraviolet exposure due to auxochromes. In case of KE lignin, the highest sun protection factor found could be related to the highest content of methoxyl and carbonyl groups as well as the highest content of ether

Table 3 SPF Values of the pure cream blended with organosolv and kraft lignins from spruce and eucalyptus

SPF

\begin{tabular}{lll}
\hline & $1 \%$ & $5 \%$ \\
\hline OS & $11.3 \pm 0.7$ & $18.5 \pm 1.2$ \\
KS & $10.6 \pm 0.7$ & $12.2 \pm 0.5$ \\
OE & $11.2 \pm 1.1$ & $14.6 \pm 0.5$ \\
KE & $12.0 \pm 0.8$ & $19.7 \pm 0.1$ \\
Cream-D & $0.8 \pm 0.0$ & \\
Cream SPF 20 & $26.9 \pm 0.7$ &
\end{tabular}




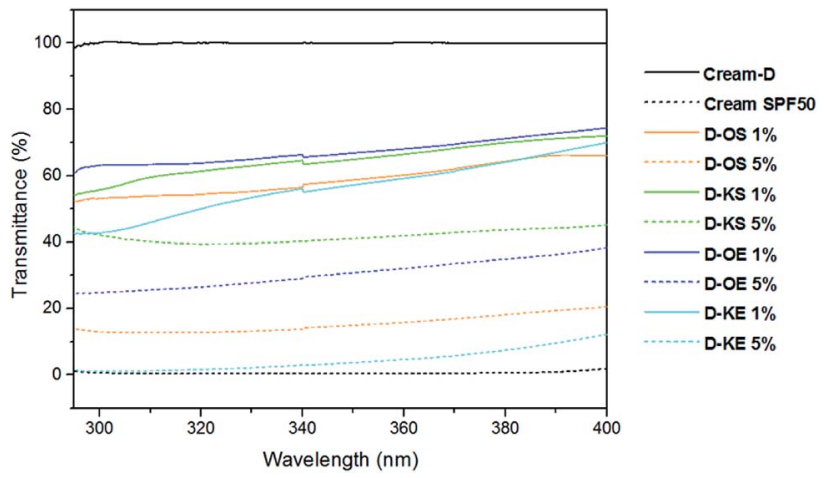

Fig. 4 UV transmittance of creams containing $1 \%$ and $5 \%$ of organosolv and kraft lignins.

linkages ${ }^{23}$ in its structure. On the other hand, a high degree of condensation in lignins often causes a negative effect as sun blocker product. ${ }^{17}$ Therefore, KS presented the lower SPF due to the highest content of condensed structures, in addition to having the lowest amount of ether bonds compared to the other lignin samples as was demonstrated in a previous work. ${ }^{23}$

With regards to the creams visual appearance, different solubility of lignins was appreciated. Kraft lignins presented better solubility than organosolv lignins; moreover the origin had some influence, being more soluble hardwood lignins. Interesting relation between SPF values of creams and $L^{*}$ parameter of lignins was found, in which higher SPF values were exhibited in darker lignins, being the trend more noticeable in creams with $5 \%$ of lignin as can be observed in Fig. 5 . Consequently, the lightness of lignin had a big influence on sunscreen property of lignin samples. Due to lignin color, the formulated lignin sunscreen lotions exhibited a light brown to brown color as its content increased providing a tanned look to the skin with their application besides being protected against solar UV radiations. Therefore, lignin would be an ideal candidate for using as natural active sunscreen against UV radiation.

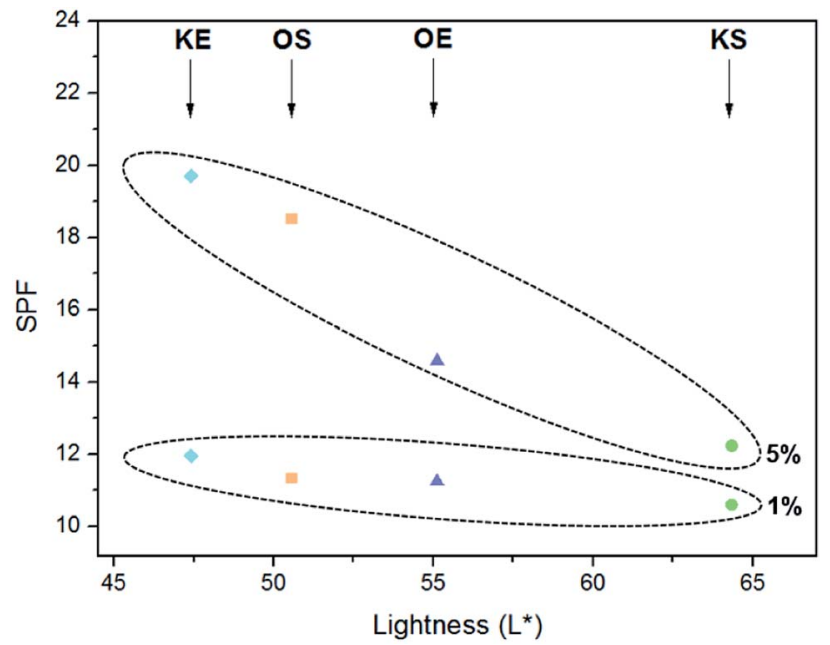

Fig. 5 SPF vs. $L^{*}$ parameter of lignins at $[1 \%]$ and $[5 \%]$ in standard commercial cream.

\section{Conclusions}

The evaluation of functional properties of lignin samples revealed that the antioxidant behaviour of lignins makes them suitable for use in cosmetic and topical formulations showing similar values to those found for commercial antioxidants. Moreover, the remarkable antimicrobial capacity of lignins, especially in the case kraft lignins, against $A$. niger and diverse food borne and human pathogenic microorganisms opens new perspectives for the food and pharmaceutical industries. The natural sunscreen power of lignins was also observed by an in vitro SPF method, suggesting their potential as additives to replace the use of typical harmful commercial chemical and physical sunscreen products. Therefore, this work demonstrated the high potential usage of lignin as an eco-friendly feedstock for several industries, thus opening innovative revalorization routes which could improve the economic profit of the biomass industry.

\section{Conflicts of interest}

There are no conflicts to declare.

\section{Acknowledgements}

The authors are grateful for the financial support received from the University of the Basque Country (doctoral grant of Ms Gordobil Grant No. PIF 13/050) and to the Basque Government (scholarship of young researchers training and project IT100816).

\section{References}

$1 \mathrm{H}$. Hatakeyama and T. Hatakeyama, in Biopolymers, ed. A. Abe, K. Dušek and S. Kobayashi, Springer-Verlag Berlin Heidelberg, 2010, vol. 232, pp. 1-63.

2 X. Pan, J. F. Kadla, K. Ehara, N. Gilkes and J. N. Saddler, Organosolv ethanol lignin from hybrid poplar as a radical scavenger: relationship between lignin structure, extraction conditions, and antioxidant activity, J. Agric. Food Chem., 2006, 54, 5806-5813.

3 Q. Lu, W. Liu, L. Yang, Y. Zu, B. Zu, M. Zhu, Y. Zhang, X. Zhang, R. Zhang, Z. Sun, J. Huang, X. Zhang and W. Li, Investigation of the effects of different organosolv pulping methods on antioxidant capacity and extraction efficiency of lignin, Food Chem., 2012, 131, 313-317.

$4 \mathrm{~J}$. H. Lora, Industrial commercial lignins: Sources, properties and applications, Monomers, Polym. Compos. from Renew. Resour., 2008, pp. 225-241.

5 M. F. Li, S. N. Sun, F. Xu and R. C. Sun, Mild acetosolv process to fractionate bamboo for the biorefinery: structural and antioxidant properties of the dissolved lignin, J. Agric. Food Chem., 2012, 60, 1703-1712.

6 G. Cazacu, M. Capraru and V. I. Popa, Advances concerning lignin utilization in new materials, Adv. Struct. Mater., 2013, 18, 255-312. 
7 W. O. S. Doherty, P. Mousavioun and C. M. Fellows, Ind. Crops Prod., 2011, 33, 259-276.

8 L. An, G. Wang, H. Jia, C. Liu, W. Sui and C. Si, Fractionation of enzymatic hydrolysis lignin by sequential extraction for enhancing antioxidant performance, Int. J. Biol. Macromol., 2017, 99, 674-681.

9 M. P. Vinardell, V. Ugartondo and M. Mitjans, Potential applications of antioxidant lignins from different sources, Ind. Crops Prod., 2008, 27, 220-223.

10 C. Pouteau, P. Dole, B. Cathala, L. Averous and N. Boquillon, Antioxidant properties of lignin in polypropylene, Polym. Degrad. Stab., 2003, 81, 9-18.

11 R. Kaur and S. K. Uppal, Structural characterization and antioxidant activity of lignin from sugarcane bagasse, Colloid Polym. Sci., 2015, 293, 2585-2592.

12 J. L. Espinoza-Acosta, P. I. Torres-Chávez, B. Ramírez-Wong, C. M. López-Saiz and B. Montaño-Leyva, Antioxidant, antimicrobial, and antimutagenic properties of technical lignins and their applications, BioResources, 2016, 11, 5452-5481.

13 M. Carocho and I. C. F. R. Ferreira, Food Chem. Toxicol., 2013, 51, 15-25.

14 H. J. Altmann, W. Grunow, P. W. Wester and U. Mohr, Induction of forestomach lesions by butylhydroxyanisole and structurally related substances, Arch. Toxicol., Suppl., 1985, 8, 114-116.

15 H. C. Grice, Safety evaluation of butylated hydroxyanisole from the perspective of effects on forestomach and oesophageal squamous epithelium, Food Chem. Toxicol., 1988, 26, 717-723.

16 J. Zemek, B. Košíková, J. Augustín and D. Joniak, Antibiotic properties of lignin components, Folia Microbiol (Praha), 1979, 24, 483-486.

17 J. M. Gutiérrez-Hernández, A. Escalante, R. N. MurilloVázquez, E. Delgado, F. J. González and G. Toríz, Use of Agave tequilana-lignin and zinc oxide nanoparticles for skin photoprotection, J. Photochem. Photobiol., B, 2016, 163, 156-161.

$18 \mathrm{~S}$. Saraf and C. Kaur, In vitro sun protection factor determination of herbal oils used in cosmetics, Pharmacogn. Res., 2010, 2, 22.

19 Y. Qian, X. Zhong, Y. Li and X. Qiu, Fabrication of uniform lignin colloidal spheres for developing natural broadspectrum sunscreens with high sun protection factor, Ind. Crops Prod., 2017, 101, 54-60.

20 A. K. Mishra, A. Mishra and P. Chattopadhyay, Assessment of In Vitro Sun Protection Factor of Calendula Officinalis L. (Asteraceae) Essential Oil Formulation, J. Young Pharm., 2012, 4, 17-21.

21 Y. Qian, X. Qiu and S. Zhu, Lignin: a nature-inspired sun blocker for broad-spectrum sunscreens, Green Chem., 2015, 17, 320-324.

22 C. Malsawmtluangi, D. K. Nath, I. Jamatia, E. Z. Lianhimgthangi and L. Pachuau, Determination of Sun Protection Factor (SPF) number of some aqueous herbal extracts, J. Appl. Pharm. Sci., 2013, 3, 150-151.
23 O. Gordobil, R. Moriana, L. Zhang, J. Labidi and O. Sevastyanova, Assesment of technical lignins for uses in biofuels and biomaterials: Structure-related properties, proximate analysis and chemical modification, Ind. Crops Prod., 2016, 83, 155-165.

$24 \mathrm{~W}$. Brand-Williams, M. E. Cuvelier and C. Berset, Use of a free radical method to evaluate antioxidant activity, Lebensm.-Wiss. Technol., 1995, 30, 25-30.

25 E. Robles, A. M. Salaberria, R. Herrera, S. C. M. Fernandes and J. Labidi, Self-bonded composite films based on cellulose nanofibers and chitin nanocrystals as antifungal materials, Carbohydr. Polym., 2016, 144, 41-49.

26 R. M. Sayre, P. P. Agin, G. J. Le Vee and E. Marlowe, A Comparison of In vivo and In vitro Testing of Sunscreening Formulas, J. Photochem. Photobiol., B, 1979, 29, 559-566.

27 R. Amorati and L. Valgimigli, Advantages and limitations of common testing methods for antioxidants, Free Radical Res., 2015, 49, 633-649.

28 N. El Abed, B. Kaabi, M. I. Smaali, M. Chabbouh, K. Habibi, M. Mejri, M. N. Marzouki and S. Ben Hadj Ahmed, Chemical composition, antioxidant and antimicrobial activities of thymus capitata essential oil with its preservative effect against listeria monocytogenes inoculated in minced beef meat, J. Evidence-Based Complementary Altern. Med., 2014, 152487, 11 pages.

29 M. Wang, J. Li, M. Rangarajan, Y. Shao, E. J. LaVoie, T.-C. Huang and C.-T. Ho, Antioxidative Phenolic Compounds from Sage (Salvia officinalis), J. Agric. Food Chem., 1998, 46, 4869-4873.

30 T. Dizhbite, G. Telysheva, V. Jurkjane and U. Viesturs, Characterization of the radical scavenging activity of lignins - natural antioxidants, Bioresour. Technol., 2004, 95, 309-317.

31 M. Lauberts, O. Sevastyanova, J. Ponomarenko, T. Dizhbite, G. Dobele, A. Volperts, L. Lauberte and G. Telysheva, Fractionation of technical lignin with ionic liquids as a method for improving purity and antioxidant activity, Ind. Crops Prod., 2017, 95, 512-520.

32 C. G. Boeriu, D. Bravo, R. J. A. Gosselink and J. E. G. Van Dam, Characterisation of structure-dependent functional properties of lignin with infrared spectroscopy, Ind. Crops Prod., 2004, 20, 205-218.

33 E. Sláviková and B. Košíková, Inhibitory effect of lignin byproducts of pulping on yeast growth, Folia Microbiol., 1994, 39, 241-243.

34 J. Zemek, M. Valent, M. Pódová, B. Kosíková and D. Joniak, Antimicrobial properties of aromatic compounds of plant origin, Folia Microbiol (Praha), 1987, 32, 421-425.

35 J. I. Pitt and A. D. Hocking, in Fungi and Food Spoilage, ed. J. I. Pitt and A. D. Hocking, Springer-Verlag, US, 3rd edn, 2009, pp. 53-143.

36 M. S. Barber, V. S. McConnell and B. S. Decaux, Antimicrobial intermediates of the general phenylpropanoid and lignin specific pathways, Phytochemistry, 2000, 54, 53-56. 
37 D. Wu, D. Lin, J. Zhang, W. Zhou, M. Zhang, Y. Zhang, D. Wang and B. Lin, Selective localization of nanofillers: Effect on morphology and crystallization of PLA/PCL blends, Macromol. Chem. Phys., 2011, 212, 613-626.

38 Y. Qian, X. Qiu and S. Zhu, Sunscreen performance of lignin from different technical resources and their general synergistic effect with synthetic sunscreens, ACS Sustainable Chem. Eng., 2016, 4, 4029-4035.
39 K. Toh, S. Nakano, H. Yokoyama, K. Ebe, K. Gotoh and H. Noda, Anti-deterioration Effect of Lignin as an Ultraviolet Absorbent in Polypropylene and Polyethylene, Polym. J., 2005, 37, 633-635.

40 S. I. Falkehag, J. MArton and E. Adler, in Lignin Structure and Reactions, ed. J. Marton, American Chemical Society, 1966, pp. 75-89. 